\title{
Micronutrient status and intervention programs in Malaysia
}

\begin{abstract}
Approximately $70 \%$ of the world's malnourished children live in Asia, giving that region the highest concentration of childhood malnutrition worldwide. Prevalence of stunting and underweight are high especially in south Asia where one in every two preschool children is stunted. Iron-deficiency anemia affects $40 \%-50 \%$ of preschool and primary schoolchildren. Nearly half of all vitamin A deficiency and xerophthalmia in the world occurs in south and southeast Asia. Iodine deficiency disorders have resulted in high goiter rates in India, Pakistan, and parts of Indonesia. Compared with other developing countries in Asia, the nutrition situation in Malaysia is considerably better, owing to rapid economic and socioeconomic development that has occurred since Malaysia gained its independence in 1957. Prevalence of undernutrition and micronutrient deficiency is markedly lower in Malaysian children. Nonetheless, undernutrition in the form of underweight, stunting, and anemia can be found in poor communities throughout the country. A prevalence of $25 \%$ underweight and $35 \%$ stunting is reported among young children from poor rural households. Anemia and subclinical forms of vitamin A deficiency were reported in children under 5 years old. Typical of a country in nutrition transition, Malaysia faces the dual burden of malnutrition in children, with the persistence of undernutrition problems especially among the poor and the emerging overweight problem especially in urban areas. Since 1996, nutrition programs of the government sector are coordinated under the National Plan of Action for Nutrition. These activities and other nutrition intervention efforts by other agencies are discussed in this paper.
\end{abstract}

Keyword: Children; Nutrition status; Intervention programs; Malaysia 